\title{
The Application of the Diels-Alder Reaction to Polymer Syntheses Based on Furan/Maleimide Reversible Couplings
}

\author{
Alessandro Gandini \\ Chemistry Departament, University of Aveiro, Portugal
}

\begin{abstract}
The furan heterocycle is a dienic reagent particularly suitable for the Diels-Alder reaction and maleimides represent a typical family of complementary reagents because of their strong dienophilic character. This paper reviews critically the studies devoted to the exploitation of the Diels Alder reaction between those moieties to synthesise macromolecular materials possessing different structures and properties. The most relevant approaches in this field are (i) polycondensation reactions calling upon A-A and B-B monomers and (ii) reversible cross-linking of linear polymers bearing pendant furan or maleimide moieties, based on the temperature sensitivity of the Diels-Alder equilibrium.
\end{abstract}

Keywords: Diels-Alder reaction, furan polymers, maleimides, polycondensation, reversible cross-linking.

\section{Introduction}

Furan chemistry is a vast domain, but its industrial aspects are essentially based on the exploitation of furfural (F) and its derivatives, since the former is readily and cheaply prepared from ubiquitous renewable resources in the form of agricultural and forestry by-products ${ }^{[1]}$.<smiles>O=Cc1ccco1</smiles>

The field of furan polymers, which was thoroughly reviewed in recent years ${ }^{[1,2]}$, represents an area of macromolecular science and technology which stands as a counterpart to petroleum-based materials, because of both the potential availability of the monomer precursors in any country and their renewable character.

The furan heterocycle differs substantially in its chemical behaviour, compared with the thiophene and pyrrole homologues, because of its pronounced dienic properties. One of the consequences of this structural feature is that furan and many of its derivatives are ideally suited dienic reagents for the Diels-Alder (DA) coupling reaction, to the point of being given as typical examples in organic chemistry textbooks, typically with maleic anhydride as the dienophile, viz.:
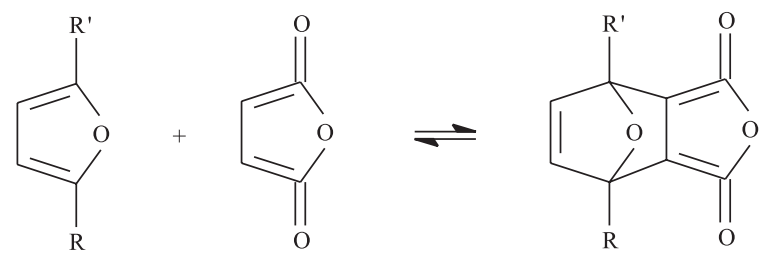

Maleimides are another family of dienophiles, which have been thoroughly studied because of their high reactivity and wide structural variability through the nature of the nitrogen substituents, providing a variety of both monofuntional and polyfunctional derivatives.

One of the most relevant aspects of the DA reaction is the high sensitivity to temperature of its equilibrium, which implies that the adducts can be readily reverted to their precursors by heating, a process known as the retro-DielsAlder (RDA) reaction.

The application of these reactions to macromolecular synthesis has received much attention in the last few decades, but the most fruitful results stem from the more recent investigations. Two distinct approaches dominate this field, namely (i) the construction of linear or cross-linked polymers through successive DA coupling reactions involving multifunctional complementary monomers, e.g. a di- or tri-furan derivative and a bismaleimide; and (ii) the formation of macromolecular networks from linear polymers bearing pendant furan and/or maleimide. In both instances, these polymers can revert to their precursors through the RDA reaction and this feature can be exploited in many applications, like the possibility of recycling or "mending" network-based materials.

The purpose of this review is to examine critically the best contributions published recently in these two areas.

\section{Step-growth reactions}

\section{Linear polymers from $A-A+B-B$ monomers}

The first studies in this context were carried out by Sastri's group ${ }^{[3,4]}$ and dealt with systems involving different difuran compounds and bismaleimides bearing aliphatic, aromatic and dimethylsiloxane spacers. These polycondensations gave products with molecular weights 
ranging from 10000 to 50000 (the latter values seem exceedingly high for this type of polymer synthesis, as discussed below), except when the difuran monomer contained silicon atoms linked directly to the $\mathrm{C} 2$ positions of the heterocyle. In that case, the DA reaction did not take place because of the electron-withdrawing effect of the $\mathrm{Si}$ atoms. The purpose of these studies was to prepare heatresistant polymers and therefore the authors did not study the RDA behaviour of the polymers, but converted instead the DA adducts along their chains into stable structures by aromatisation, viz.:

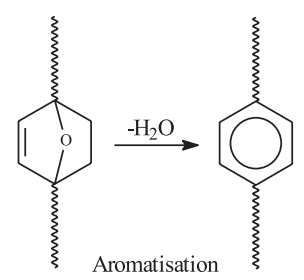

O'Dell followed shortly thereafter with a thorough investigation of the DA polycondensation of various difuran derivatives with classical bismaleimides, which unfortunately was not published in a journal format ${ }^{[5]}$. Multiply substituted furans failed to give high DP because of steric hindrance in the formation of the adducts, whereas unsubstituted difurans linked through both $\mathrm{C} 2$ positions gave better results with DPs often higher than 20. A careful study of the polymer structure confirmed that the chain growth had indeed taken place through successive DA couplings, giving a majority of exo structures. The author's study of the aromatisation of the adduct moieties clearly showed that this reaction was less straightforward and its yields much lower than previously claimed without convincing evidence.

A series of papers published in the early ninetie ${ }^{[6-8]}$ dealt with systems involving the DA polymerisation between a difuran derivative and a bismaileimide. These studies did not focus on a detailed structural analysis of the polymer and contributed only modestly to the advancement of the topic with respect to the investigations reported earlier, except for the observation of (partial) reversibility at $90{ }^{\circ} \mathrm{C}$ in the case of a polymer prepared from di-2-furfuryl adipate and bismaleimidodiphenylmethane ${ }^{[6]}$.

In more recent years, we carried out a systematic study of the DA polymerisation involving a difuran diacetal (F-F) and either an aliphatic or an aromatic bismaleimide (M-M) ${ }^{[9]}$. Model compounds were first prepared from, respectively, both monofunctional complementaly homologues ( $\mathrm{F}+\mathrm{M}$ dimers $)$ and from the different combinations of monofunctional reagents with the complementary difunctional counterpart (F-F $+\mathrm{M}$ and $\mathrm{F}+\mathrm{M}-\mathrm{M})$. The structures of these model adducts were carefully assessed, as were the proportions of exo and endo isomers. With this basic information, the actual polymerisation could be carried out in the most adequate conditions and the structure of the ensuing materials assessed. The following mechanism applied to the aromatic bismaleimide:
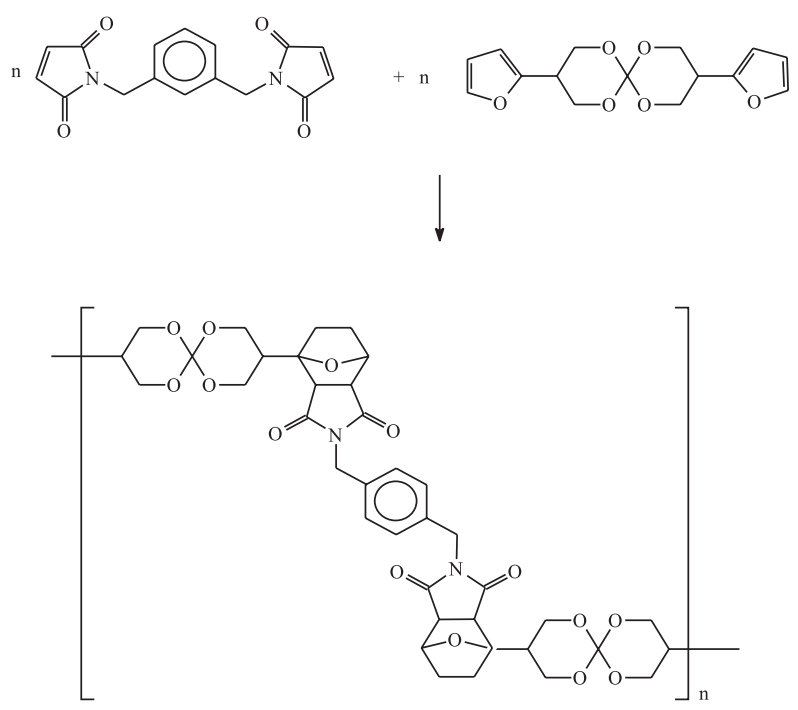

The ${ }^{1} \mathrm{H}$ NMR spectra of the polymers suggested that chain growth had not been perturbed by detectable side reactions. However, the early precipitation of the polymers from the reaction medium limited their DP to a few tens.

A similar study ${ }^{[10]}$ was conducted using much more flexible monomer structures in order to enhance polymer solubility, viz.:

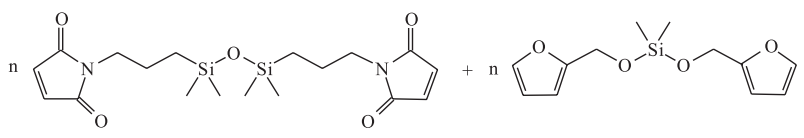

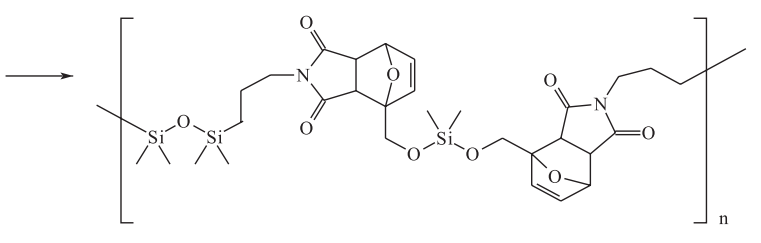

(57)

and indeed higher DPs were obtained.

Kamahori et al. ${ }^{[11]}$ prepared the same type of polymers using chiral Lewis acids as catalysts and obtained optically active products with DPs of about 20.

\section{Linear polymers from $A-B$ monomers}

The first attempts to prepare a molecular structure bearing both a furan and a maleimide moiety ${ }^{[12,13]}$ were not corroborated by a convincing structural analysis. It is likely that the conditions used for their synthesis were too severe and the expected products resinified before isolation.

We tackled this issue using milder conditions and obtained 2-furfurylmaleimide (FM) from 2-furfuryl amine and maleic anhydride by the classical two-step synthesis, going through the corresponding N-2-furfurylmaleamic acid.

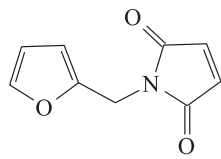

FM 
FM is a crystalline compound, m.p. $106^{\circ} \mathrm{C}$, which gave the correct elemental analysis and the expected mass, FTIR, ${ }^{1} \mathrm{H}$ and ${ }^{13} \mathrm{C}$ NMR spectra. It displayed a high sensitivity to moisture, which induced its ring-opening hydrolysis regenerating the maleamic acid.

The polymerisation of FM was studied in sealed tubes both in bulk and in 1,1,2,2-tetrachloroethane at temperatures above $150^{\circ} \mathrm{C}$ and gave partially soluble products, suggesting that the high temperatures necessary to induce its self-condensation through the DA reaction were also responsible for some aromatisation of the adducts. The soluble fraction, however, gave a ${ }^{1} \mathrm{H}$ NMR spectrum indicating that regular DA oligomers had indeed been formed according to the following mechanism:

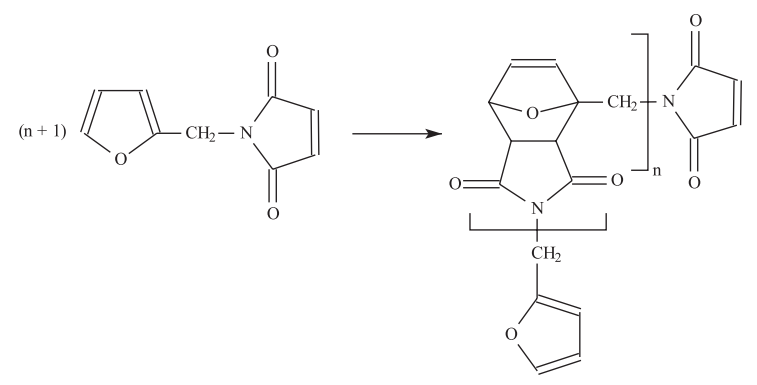

The study of A-B monomers is still in progress.

\section{Dendrimers and networks}

The first (and hitherto the only) application of the DA reaction to prepare thermally responsive dendrons and dendrimers ${ }^{[15]}$ is a very original piece of work based on a furan $\mathrm{AB}_{2}$ monomer with aromatic ether moieties at $\mathrm{C} 3$ and $\mathrm{C} 4$ in conjunction with a maleimide bearing a reactive phenolic moiety. The third generation dendrimer (MW 5469.10) was successfully constructed and had the following structure:

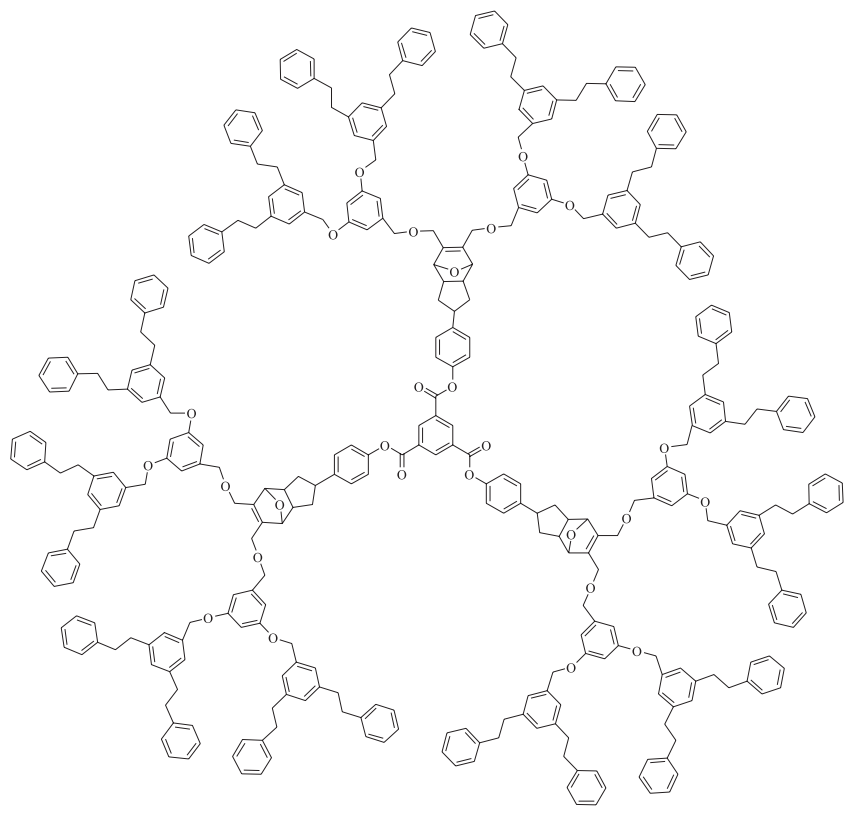

These products were heated at $110{ }^{\circ} \mathrm{C}$ in DMF- $d_{7}$ in a NMR tube and the DA-RDA equilibrium was reached within an hour to give $40 \%$ dissociation of the adduct sites. Upon cooling to $65^{\circ} \mathrm{C}$ the dendrimers were fully reconstituted, albeit in several days.

This nice piece of work constitutes the first example of a thermally labile-reassembling dendrimer exploiting the DARDA approach.

In another vein, Wudl's group applied the DA-RDA strategy to "mendable" polymers ${ }^{[16,17]}$. Different networks, all based on the DA polycondensation between multifunctional furans and maleimides, were prepared and characterised. Scheme 1 shows the synthesis of one such polymer. These clear solid products were submitted to heating/freezing cycles and their structural changes followed by solid-state ${ }^{13} \mathrm{C}$ NMR spectroscopy. The RDA reaction occurred inside the network at $\sim 120^{\circ} \mathrm{C}$ and heating the annealed polymers at $80-90{ }^{\circ} \mathrm{C}$, viz. well above their $\mathrm{Tg}$, and then cooling them to room temperature, reconstituted, through the DA reaction, the thermally-dissociated adducts. Cracks were induced in these materials, which were first submitted to a heating treatment to heal them and then slowly cooled to room temperature to reconstitute the heat-opened adducts. It was shown in this way that the DA/RDA principle provides a simple and efficient way to prepare mendable polymers.
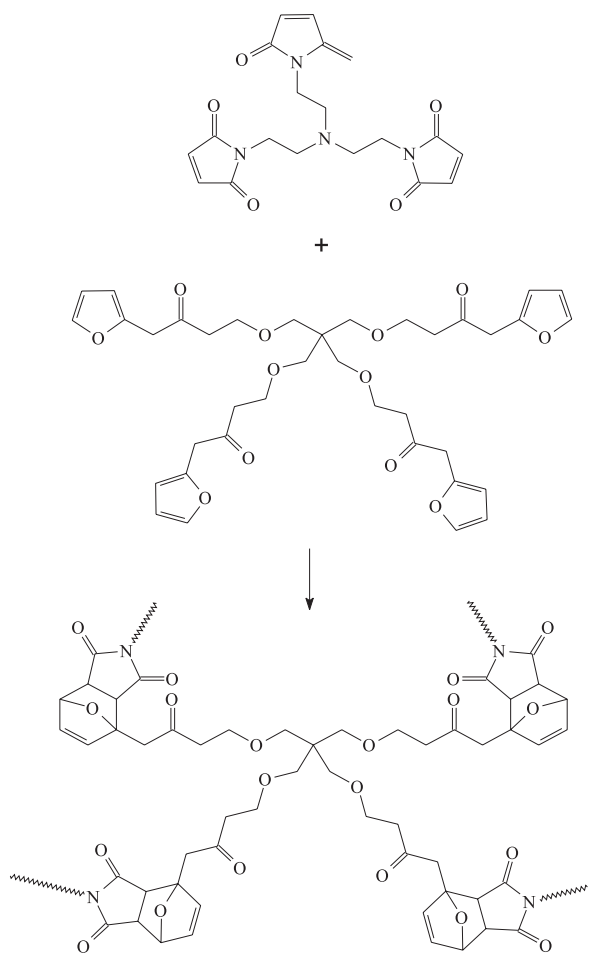

$(60)$

Scheme 1

\section{Reversible cross-linking reactions}

An early study dealing with the possibility of synthesizing thermally reversible networks from linear polymers bearing reactive DA functions describes the reaction of difurans with maleimide-substituted polymers ${ }^{[18]}$. It was however only starting from the nineties that this research topic gained momentum. 
Saegusa's group published a study ${ }^{[19]}$ describing the reversible cross-linking of modified poly(N-acetylethyleneimine)s bearing either maleimide or furancarbonyl pending moieties. The authors claimed that mixing the two modified complementary polymers resulted in a highly crosslinked material and that heating this gel at $80^{\circ} \mathrm{C}$ for two hours in a polar solvent, regenerated the two starting linear precursors in $100 \%$ yield. These results are surprising for two reasons, namely: on the one hand, furancarbonyl moieties are known not to be prone to intervene the DA reaction and, on the other hand, it is difficult to understand why the two polymers, separated at high temperature by the RDA reaction, did not recombine, even in part, through the DA reaction, when their solution was cooled at room temperature. Moreover, $80^{\circ} \mathrm{C}$ seems a relatively low temperature to insure $100 \%$ RDA decoupling, instead of an equilibrium situation, as well documented in both model and polymer reactions ${ }^{[15,17,20]}$. The same reservations apply to a later study of the same system ${ }^{[21]}$.

Canary and Stevens ${ }^{[20]}$ described the thermally reversible cross-linking of polystyrene bearing maleimide side groups with di-2-furfuryl adipate and reported that the RDA reaction was particularly effective at $150{ }^{\circ} \mathrm{C}$, but also noticed that the thermal instability of the furfuryl moiety was a serious limitation to the viability of their system. We confirmed the latter drawback in a preliminary study of the DA/RDA reaction of N,N'-methylenediphenylbismaleimide (MDPBM) with random copolymers of methyl methacrylate with 2furfuryl acrylate ${ }^{[22]}$. Crosslinking readily occurred in solution, but the gels did not revert entirely by the RDA reaction in bulk (TGA) and the residual proportion of network was the higher, the higher the furan content in the initial copolymer. With 10 moles $\%$ of heterocycles, $20 \%$ by weight of un-crosslinked residue was left after heating. This problem is associated with the formation of free radicals arising from the methylene group attached to the $\mathrm{C} 2$ position in the furfuryl moiety ${ }^{[2]}$ and can be minimised by using an inert atmosphere and adding a radical inhibitor to the substrate.

To the best of my knowledge, the first systematic approach to a reversible DA/RDA cross-linking system was carried out with styrene copolymers bearing different proportions of pendant 2-furfuryloxy moieties in conjunction with $\operatorname{MDPBM}^{[23]}$. The copolymers were first submitted to a model reaction with the monofunctional N-phenylmaleimide, in order to study the DA/RDA cycle without the complication of gel formation (left-hand side of Scheme 2). The DA reaction proceeded smoothly in refluxing methylene chloride. The ensuing copolymers bearing the adducts were dissolved in a $80 / 20 \mathrm{v} / \mathrm{v}$ mixture of chlorobenzene/2-methylfuran and the solution brought to reflux for $24-48 \mathrm{~h}$ (depending on the percentage of adduct in the copolymer).

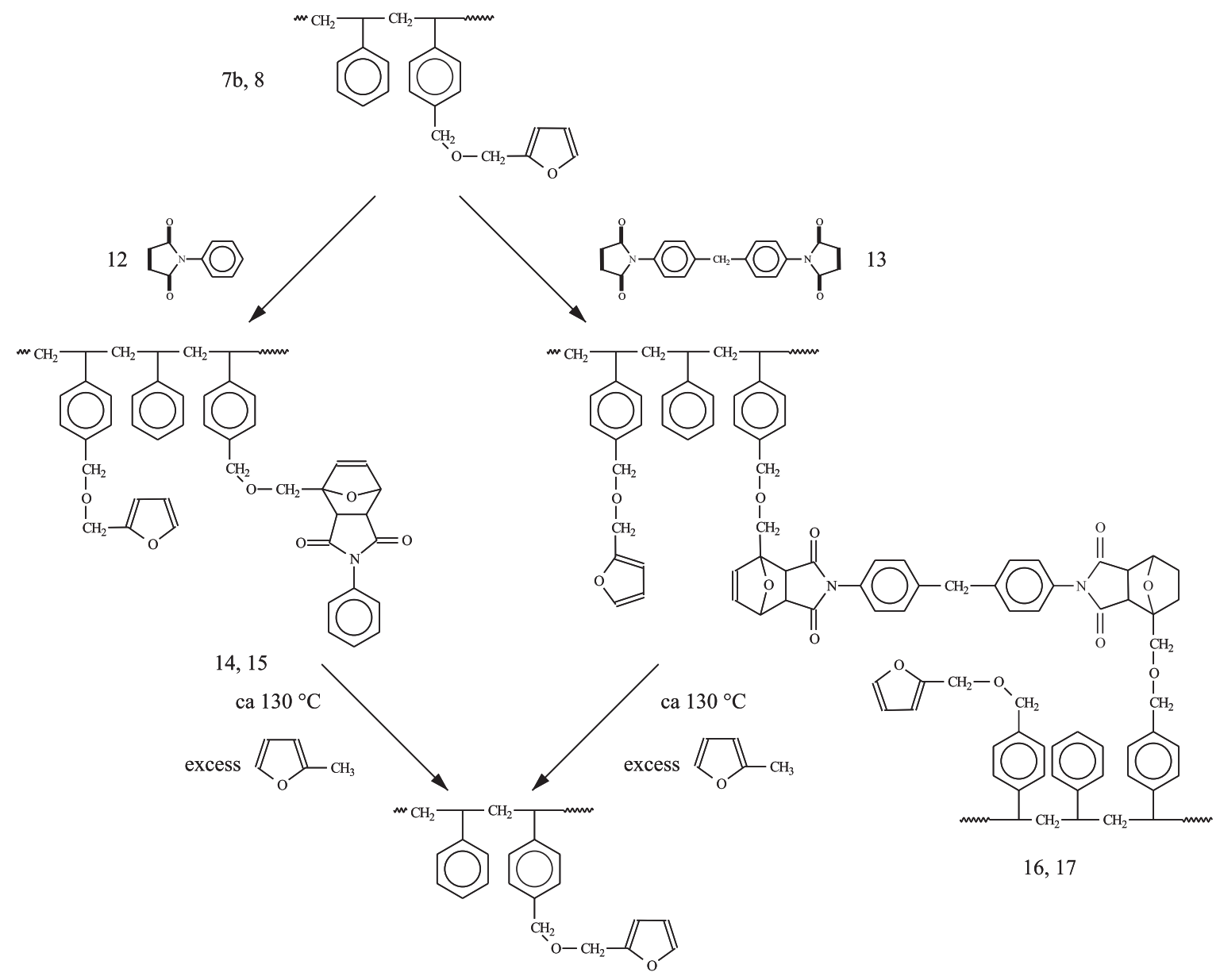

Scheme 2 
The original styrene-furan copolymers were recovered quantitatively after cooling to room temperature, together with the DA adduct of N-phenylmaleimide with 2-methylfuran. The presence of a large excess of 2-methylfuran (with respect to the furan moieties appended to the copolymers) during the RDA reaction and while cooling the ensuing solution, insured the trapping of all the released maleimide.

The same strategy was then applied to the crosslinking and de-cross-linking of the copolymers with MDPBM (righthand side of Scheme 2). The DA reaction produced the expected gelled materials, which were characterised and then submitted to the RDA reaction in the presence of a large excess of 2-methylfuran. The occurrence of de-cross-linking was clearly suggested by the progressive dissolution of the gels and within a few hours all the suspended materials had vanished. The precipitated polymers showed all the structural features of the corresponding initial copolymers, suggesting that the RDA reaction in the presence of the trapping agent had gone to completion. This was confirmed by the recovery of the DA bisadduct between 2-methylfuran and MDPBM. Thus, at a temperature of $\sim 130{ }^{\circ} \mathrm{C}$ and with the addition of a trap, the DA/RDA cycle was optimised and side reactions related to the furfuryl moieties avoided.

Further studies in this context concerned copolymer with elastomeric properties ${ }^{[10,24,25]}$. One system followed the same pattern as the original study ${ }^{[23]}$, but the furan acrylic copolymer had Tg lower than $-30{ }^{\circ} \mathrm{C}$ and the bismaleimide used for their cross-linking had an oligoether spacing which gave it a high flexibility $\left(\mathrm{Tg} \sim-110^{\circ} \mathrm{C}\right)$. Scheme 3 illustrates this system:<smiles>COC(=O)C(CC(C)C)CC(C)C(=O)Oc1ccco1</smiles>

(66)

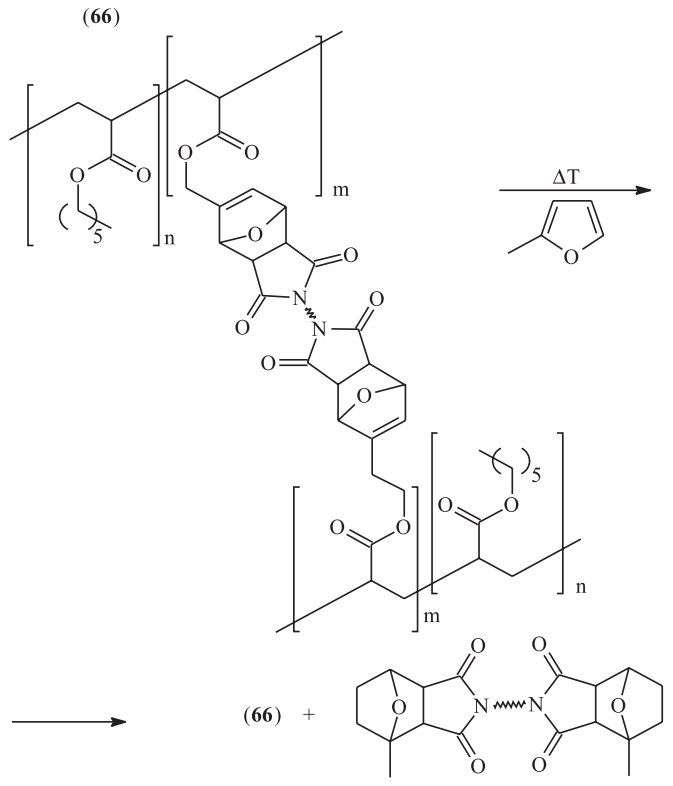

Scheme 3
The second system was based on the reverse approach, namely the reaction of an elastomeric polydimethylsiloxane (PDMS) copolymer bearing pendant maleimide moieties with a flexible difuran derivative, as shown in Scheme 4:

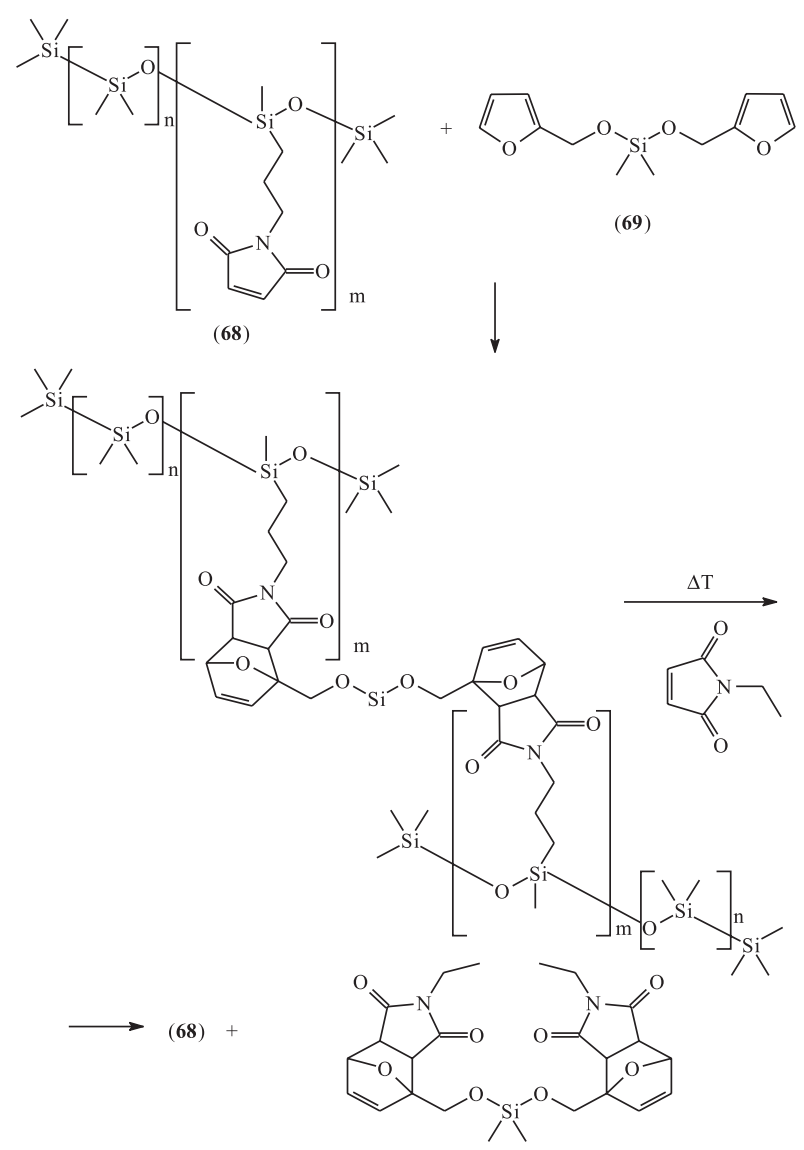

Scheme 4

In both instances, the DA/RDA cycles were optimised by the use of appropriate traps, as shown in Schemes 3 and 4. Two further pieces of evidence were obtained in these studies, concerning the temperature at which the RDA reaction becomes relevant. Figure 1 shows the dynamic mechanical behaviour of one of the cross-linked acrylic copolymers.

After the characteristic drop of the elastic modulus at $\sim-40{ }^{\circ} \mathrm{C}$ (Tg of the network), the elastic behaviour extends up to $\sim 90^{\circ} \mathrm{C}$, where the RDA reaction begins and the ensuing linear chains display a drop in E' associated with viscous flow.

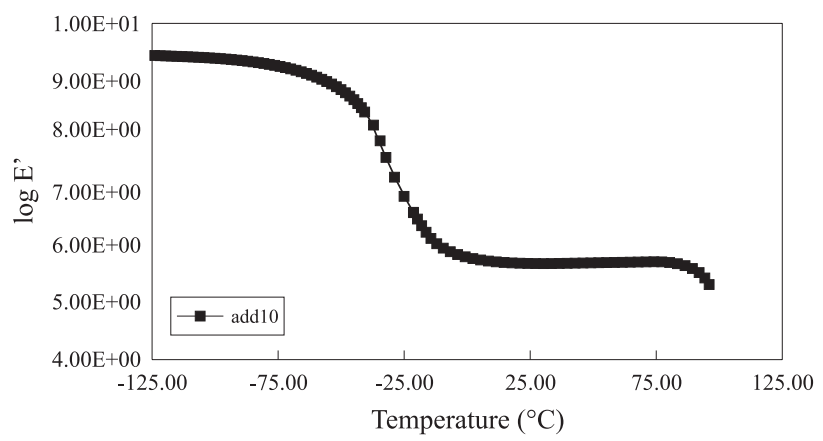

Figure 1 


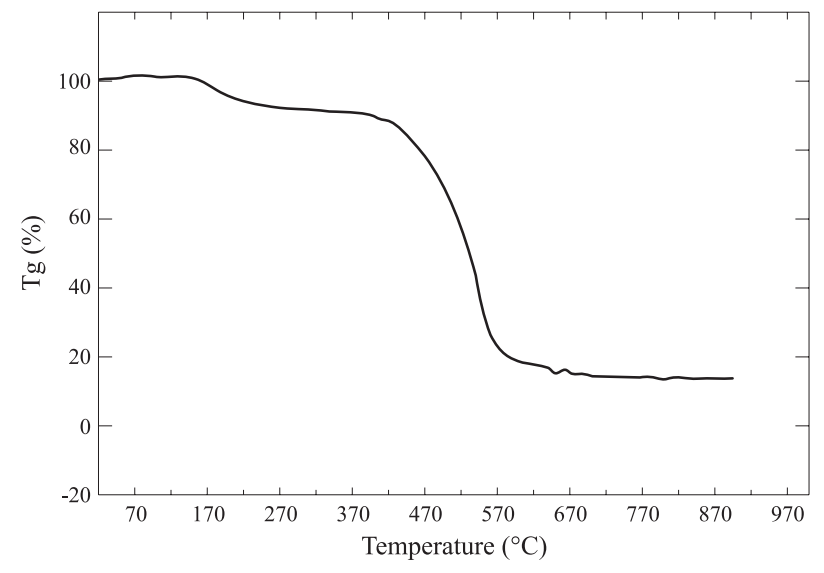

Figure 2.

Figure 2 shows the TGA tracing of one of the PDMS networks.

The weight loss between 140 and $300{ }^{\circ} \mathrm{C}$ corresponds to the progressive release and evaporation of the difuran crossliker (Scheme 4), which was calculated to correspond closely to its total amount in the analysed sample.

In both these studies ${ }^{[10,25]}$, the swelling kinetics and equilibria in various media of the networks were investigated in order to determine their cross-link densities and compare them with those obtained from dynamic-mechanical measurements.

One of the promising consequence of these studies is the application of their concepts to the recycling of tyres, an industrial and ecological problem which has found no viable solution to date.

Huglin's group recently investigated a DA/RDA system using copolymers, which were essentially the same as those shown in Scheme 3 (except that their Tg were above room temperature) and MDPBM as the cross-linker ${ }^{[26-29]}$. The interest of these contributions resides, on the one hand, in a careful study of swelling and mechanical properties of the networks ${ }^{[28]}$ and, on the other hand, in an investigation of the kinetics of both the DA cross-linking ${ }^{[29]}$ and the RDA decross-linking processes ${ }^{[27]}$. These reactions were followed by UV spectroscopy, taking advantage of the fact that the maleimide moieties absorb around $300 \mathrm{~nm}$, whereas the corresponding adducts do not, because of the loss of conjugation between the carbonyl functions associated with the disappearance of the $\mathrm{C}=\mathrm{C}$ unsaturation. Thus, the rate of maleimide consumption was monitored in the case of the DA cross-linking reaction. Conversely, the rate of its regeneration was followed spectroscopically by heating the network in different high-boiling solvents, which induced the progressive RDA de-cross-linking and the consequent solubilisation of the released bismaleimide.

\section{Conclusion}

Considerable advances have been achieved in recent years both in the understanding and the optimisation of the features related to the application of the $\mathrm{DA} / \mathrm{RDA}$ reactions to furan/ maleimide couplings for macromolecular synthesis and in the preparation of materials with a wide range of possible utilisations. The thermally reversible character of these interactions is certainly their most appealing aspect, because it provides a straightforward means of regenerating the monomeric or macromolecular reagents, thus opening the way to such materials as recyclable networks and liquid/solid phase-changing compositions.

\section{References}

1. Moreau, C.; Gandini, A.; Belgacem, M.N. - Topics Catalysis 2711 (2004).

2. Gandini, A.; Belgacem, M. N. - Prog. Polym. Sci. 22, 1203 (1997).

3. G.C. Tesoro, V.R. Sastri, Ind. Eng. Chem. Prod. Res. Dev., 25, 444 (1986).

4. He, X.; Sastri, V.R.; Tesoro, G. C. - Makromol. Chem. Rapid. Comm. 9, 191 (1989).

5. O’Dell, R. - PhD Thesis, Lancaster University, UK (1990).

6. Kuramoto, N.; Hayashi, K.; Nagai, K. - J. Polym. Sci. Polym. Chem. 30, 2501 (1992).

7. Diakoumakos, C. D.; Microyannidis, J.A. - J. Polym. Sci. Polym. Chem. 30, 2559 (1992).

8. Diakoumakos, C. D.; Microyannidis, J.A. - Eur. Poly. J. 30, 465 (1994).

9. Goussé, C.; Gandini, A. - Polym. Intern. 48, 723 (1999).

10. Pérez-Berumen, C.; Gandini, A. - unpublished results; C. Pérez-Berumen, Doctorate Thesis, National Polytechnic Institute, Grenoble, France (2003),

11. Kamahori, K.; Tada, S.; Ito, K.; Itsuno, S. Macromolecules 32, 541 (1999).

12. Mikroyannidis, J. A. - J. Polym. Sci. Polym. Chem. 30, 125 (1992).

13. Mikroyannidis, J. A. - J. Polym. Sci. Polym. Chem. 30, 2017 (1992).

14. Goussé, C.; Gandini, A. - Polym Bull. 40, 389 (1998).

15. McElhanon, J. R.; Wheeler, D.R. - Org. Lett. 3, 2681 (2001).

16. Chen, X.; Dam, M. A.; Ono, K.; Mal, A.K.; Shen, H.; Nutt, S. R.; Sheran, K.; Wudl, F. - Science, 295, 1698 (2002).

17. Chen, X.; Wudl, F.; Mal, A. K.; Shen, H.; Nutt, S. R. Macrolmolecules, 36, 1802 (2003).

18. Stevens, M.; Jenkins, A. - J. Polym. Sci, 17, 3675 (1979).

19. Chujo, Y.; Sada, K.; Saegusa, T. - Macromolecules 23, 2636 (1990). 
20. Canary, S. A.; Stevens, M. P. - J. Polym. Sci. Polym. Chem. 30, 1755 (1992).

21. Imai, Y.; Itoh, H.; Naka, K.; Chujo, Y. - Macromolecules, 33,4343 (2000).

22. Laita, H.; Boufi, S.; Gandini, A. - Eur. Polym. J. 33, 1203 (1997).

23. Goussé, C.; Gandini, A.; Hodge, P. - Macromolecules, 31, 314 (1998).

24. Gheneim, R.; Pérez-Brumen, C.; Gandini, A. Macromolecules, 35, 7246 (2002).
25. Gheneim, R. - Doctorate Thesis, National Polytechnic Institute, Grenoble, France (2003),

26. Goiti, E.; Huglin, M. B.; Rego, J. M. - Polymer, 42, 10187 (2001).

27. Goiti, E.; Huglin, M. B.; Rego, J. M. - Macromol. Rapid Comm., 24, 692 (2003).

28. Goiti, E.; Huglin, M. B.; Rego, J. M. - Eur. Polym. J. 40, 219 (2004)

29. Goiti, E.; Huglin, M. B.; Rego, J. M. - Eur. Polym. J. 40, 1451 (2004). 